\title{
ZS Research Square

\section{Resting coronary flow estimated by dynamic coronary CT angiography: Potential for use in the detection of plaque burden and myocardial ischemia}

\section{Kiyoe Ando}

Tokyo Women's Medical University

Michinobu Nagao ( $\nabla$ nagao.michinobu@twmu.ac.jp)

Tokyo Women's Medical University

Atsushi Yamamoto

Tokyo Women's Medical University

Risako Nakao

Tokyo Women's Medical University

Akiko Sakai

Tokyo Women's Medical University

Eri Watanabe

Tokyo Women's Medical University

Kayoko Sato

Tokyo Women's Medical University

Kenji Fukushima

Saitama Medical University

Shuji Sakai

Tokyo Women's Medical University

Nobuhisa Hagiwara

Tokyo Women's Medical University

\section{Research Article}

Keywords: coronary artery disease, coronary flow index, coronary CT angiography

Posted Date: February 19th, 2021

DOl: https://doi.org/10.21203/rs.3.rs-214072/v1

License: (c) (1) This work is licensed under a Creative Commons Attribution 4.0 International License. Read Full License 
Page 2/17 


\section{Abstract \\ Purpose}

We propose a resting coronary flow index ( $\mathrm{rCFI}$ ) estimated by dynamic coronary CT angiography (CCTA) for potential use in detecting coronary plaque burden and myocardial ischemia.

\section{Materials and Methods}

A total of 124 patients with stable coronary artery disease (CAD) who underwent CCTA were prospectively enrolled in this study. The original data set of dynamic CCTA with continuous 8 to 12 cardiac cycles was interpolated to 24 to 36 image sets using the motion coherence image processing. Using the maximum slope method, rCFI was defined as the ratio of the maximum upslope in the attenuation of distal coronary artery to that of the ascending aorta. Coronary plaques were classified as non-calcified or calcified based on the presence or absence of low attenuation plaque. For patients who may need additional functional assessment, additional stress/rest myocardial perfusion scintigraphy (MPS) was performed.

\section{Results}

rCFI for vessels with non-calcified plaques was significantly lower than that for normal arteries $(0.73 \pm$ 0.23 vs. $0.88 \pm 0.22, p<0.00001)$. In limited intermediate stenosis, a similar significant difference was observed between the two groups $(0.76 \pm 0.23$ vs. $0.89 \pm 0.20, p<0.0001)$. In contrast, no significant decrease in rCFI was observed between normal and calcified plaques $(0.81 \pm 0.28)$. rCFI for ischemia depicted by MPS was significantly lower than for non-ischemia $(0.63 \pm 0.24$ vs. $0.84 \pm 0.19, p<0.05)$. The use of the optimal cutoff could detect ischemia, with a C-static value of $0.73,53 \%$ sensitivity, and $93 \%$ specificity.

\section{Conclusion}

rCFI can be used for the detection of plaque burden and myocardial ischemia in CAD.

\section{Introduction}

Coronary CT angiography (CCTA) is a standard imaging technique used to detect coronary stenosis and atherosclerosis. According to the latest guidelines, functional coronary stenosis, rather than anatomic coronary stenosis, is key in determining whether to perform coronary intervention such as revascularization therapy ${ }^{1,2}$. Fractional Flow Reserve (FFR) and instantaneous wave-free ratio (iFR) are well-known indices for detecting functional coronary stenosis ${ }^{3}$. FFR and iFR are coronary pressure ratios measured by a pressure wire during invasive coronary angiography, and are used to estimate coronary 
flow based on the linear correlation between coronary pressure and flow under specific conditions 4,5 . Meanwhile, non-invasive methods for detecting functional coronary stenosis are becoming more popular, such as CT-derived FFR (FFR-CT), which estimates hyperemic coronary pressure from CCTA images using fluid mechanics, has been developed ${ }^{6,7}$. Recent studies have reported the method's high sensitivity and diagnostic ability for detecting functional stenosis compared with conventional CCTA ${ }^{6,7}$.

FFR has been the gold standard for the indication of revascularization therapy until recently, when major studies revealed the non-inferiority of iFR against FFR. iFR is expected to become the new gold standard, because of its superiority over FFR in term of causing less chest discomfort and allowing for shorter operation times. CCTA verified positive remodeling, spotty calcification, and low attenuation plaques ( $<30$ Hounsfileds units [HU]) are considered morphological characteristics of high-risk plaques (HRP), which are independent predictors of acute coronary syndrome ${ }^{8}$. However, the influence of HRP on coronary artery flow in vivo has not been elucidated. The present study proposes a resting coronary flow index (rCFI) estimated by dynamic CCTA combined with a low dose and boost scan to investigate the influence of HRP on coronary flow. In addition, we investigate the ability of rCFI in detecting myocardial ischemia, which is conventionally depicted by stress/rest myocardial perfusion scintigraphy (MPS).

\section{Materials And Methods}

\section{Study population}

The study population was comprised of 124 stable patients with suspected or known CAD who underwent CCTA between June 2017 and November 2019, prospectively. Exclusion criteria were as follows; age under 35 years, patients with allergy to iodinated contrast, renal dysfunction except for dialysis patients (estimated glomerular filtration rate $<30 \mathrm{~mL} / \mathrm{min} / 1.73 \mathrm{~m}^{2}$ ), systolic blood pressure $<90$ $\mathrm{mmHg}$, prior coronary artery bypass surgery (CABG), acute myocardial infarction (within 3 months), unstable angina (recent onset of angina within 1 month, or severe and worsening clinical symptoms), severe left ventricular dysfunction (left ventricular ejection fraction $<20 \%$ ), congestive heart failure (New York Heart Association class $\otimes$ ), significant valvular disease, non-ischemic cardiomyopathy, and congenital heart disease. Patients with high heart rate (over $70 \mathrm{bpm}$ at the time of scan) or arrhythmia including frequent extra beats were also excluded. Patient characteristics are summarized in Table 1. Stented vessels with chronic total occlusion or with diffuse circumferential advanced calcification were excluded from the analysis. Any medical history of diabetes, hypertension, and hyperlipidemia was extracted from all the patients' medical records and reviewed. Patient with a prior medical history, regardless of current treatment, were considered positive. Informed written consent was obtained from each patient before the study, and complied with the Declaration of Helsinki. This study was approved by the Ethics Committee of Tokyo Women's Medical University.

\section{Dynamic CCTA scan with dose modulation}


All participants underwent dynamic CCTA with dose modulation using a 320-row CT scanner (Aquilion One Genesis Edition; Cannon Medical Systems Co., Tochigi, Japan). This dynamic scan protocol builds based on the previous studies ${ }^{9,10}$. Intravenous or oral metoprolol $(20 \mathrm{mg})$ was administered for patients with a heart rate of $\geq 65$ beats $/ \mathrm{min}$. Immediately before image acquisition, all patients received sublingual nitroglycerine $0.2 \mathrm{mg}$. First, the test-bolus examination was performed using prospective electrocardiography (ECG)-gating axial scans. This was done at the ascending aorta, at the slice level of the left main trunk in mid-diastole for 15-25 cardiac cycles during a 20.0-second period and starting at 7.0 seconds after the initiation of the bolus injection of the contrast media to determine the optimal scan timing. After the test-bolus examination, dynamic CCTA scanning was continuously performed in middiastole for 8 to 12 cardiac cycles with prospective ECG-gating axial scans after a 10-seconds contrast media injection (259 mg/kg, lopamiron 370; Bayel Healthcare, Osaka, Japan). One scan of the dynamic CCTA was performed as a boost scan for standard CCTA at the peak phase of the ascending aorta, which was determined by a test bolus examination. The acquisition parameters are summarized in Table 2 .

The standard CCTA was analyzed using a workstation (Ziostation, Ziosoft, Tokyo, Japan) based on a combination of transverse sections and automatically generated curved multiplanar reconstruction (MPR) images of the target vessels. A semi-automated vessel analysis tool was used for grading the severity of stenosis. Images were clinically interpreted by the consensus decision of an experienced radiologist and cardiologist, using the American Heart Association (AHA) 15-segment model. The degree of coronary stenosis was classified as follows: none ( $0 \%$ ), mild ( $1 \%$ to $49 \%)$, moderate $(50 \%$ to $74 \%$ ), and severe $(\geq 75 \%)$. A single vessel having multiple lesions was considered as one with the most severe stenosis. Coronary plaques were classified as non-calcified and calcified based on the presence or absence of low attenuation plaque (LAP) and positive remodeling. LAP was defined as plaques containing any voxel $<30 \mathrm{HU}^{8}$. Positive remodeling was defined as a ratio $>1.1$ between the vessel area at the lesion and that of a proximal reference point ${ }^{8}$. Plaques with LAP, positive remodeling, and partially calcified plaques were classified as non-calcified. Completely calcified plaques without LAP and positive remodeling were defined as calcified plaques. No plaque or calcification in the target vessel was defined as normal.

We recorded the machine-generated volume CT dose index (mGy) and the dose-length product (DLP, mGy.cm) for the dynamic CCTA scan ${ }^{11}$.

\section{Calculation of rCFI}

The data set for dynamic CCTA with continuous 8 to 12 cardiac cycles was transformed into commercially available software (Ziostation 2 Phyziodynamics; Ziosoft, Tokyo, Japan). Through the motion coherence image processing (MCIP) ${ }^{9,10}$, the original data set was interpolated by neighbouring phases up to 24 to 36 dynamic image sets (Fig. 1A). In the converted dynamic series, a spherical volume of interest ( $\mathrm{VOI}$ ) was drawn in the ascending aorta and distal sites of coronary arteries with a diameter of $2 \mathrm{~mm}$ (coronary segment \#3 in the right coronary artery, \#7-8 in the left anterior descending artery, \#13-14 in the left circumflex artery, and the distal site of the major branches such as the diagonal, ramus, and 
obtuse marginal in case where significant myocardial ischemia was proven by myocardial perfusion imaging). Once VOI was activated, it automatically tracked the movement of the coronary artery, it automatically tracked with voxel in all phases ${ }^{12}$. CT value was then exported as a time-density curve in Microsoft Excel. The slope of the dynamic curve was set for its starting point and the peak. Based on the maximum slope method, rCFI was generated using the ratio of the maximum upslope in the distal coronary artery and that of the ascending aorta (Fig. 1B). In patients with coronary calcifications, VOI was placed in a manner that avoided those calcifications.

\section{Stress/rest myocardial perfusion scintigraphy (MPS)}

For patients with mild to moderate coronary stenosis, with atherosclerotic plaques on CCTA, and who may need additional functional assessment, an adenosine stress/rest ${ }^{99 \mathrm{~m}} \mathrm{Tc}$ tetrofosmin (Myoview ${ }^{\circledR}$, Nihon-mediphysics, Tokyo) single-photon emission computed tomography (SPECT) was performed. ${ }^{99 m}$ Tc SPECT images were obtained using a dual-head SPECT/CT gamma-detector system (Symbia S®, Siemens Healthcare, Erlangen, Germany) equipped with a smart zoom collimator. A total of 30 projection data sets were obtained in a $64 \times 64$ matrix over a $180^{\circ}$ arc. The acquisition time was 40 seconds after stress and 30 seconds at rest for each projection. Adenosine stress $(0.12 \mathrm{mg} / \mathrm{min} / \mathrm{kg})$ was administrated 3 minutes prior to stress scan and continued for 6 minutes. All patients were refrained from aminophylline-containing medications or caffeine-containing beverages at least 12 hours before the scan. SPECT images were reconstructed and converted to a polar map analysis by a commercially available, stand-alone automatic tool (QGS®, Ceders-sinai, California). Visual scoring using a 0 to 4-point scale was performed with a polar map output according to the AHA 17 segment model ${ }^{13}$. A summed differential score (SDS) $>2$ per each coronary territory was defined as positive for myocardial ischemia ${ }^{14,15}$. Based on each patient's MPS results, we categorized the territories of the RCA, the LAD, and the LCX as non-ischemic or ischemic. The ischemic territory was defined as a territory that consisted of one or more segments of ischemia.

\section{Statistical analysis}

Continuous data was expressed as the mean \pm the standard deviation. Testing of differences between demographic and clinical data of rCFI and other factors were accomplished using the Kruskal-Wallis test or the Mann-Whitney U test for continuous variables, and either the Pearson's chi-square test or Fisher's exact test for categorical variables. A receiver-operating-cumulative curve (ROC) analysis was performed to determine the optimal cutoff of the rCFI for the detection of ischemia, and to investigate the area under the curve (AUC), sensitivity, and specificity. All statistical tests conducted were two-sided. A $p$-value of $<0.05$ was considered statistically significant when comparing two groups. A $p$-value of $<0.016$ was considered statistically significant when comparing three groups. These analyses were performed using the JMP statistical software (Version 9.0; JMP, Inc., Cary, North Carolina).

\section{Results}


rCFI of all 124 patients were measured. Baseline characteristics are listed in Table 1. The 30 participants who had no stenosis or calcification in all their vessels were considered as normal controls. Their three major vessels were defined as normal coronary arteries. rCFI for control patients was $0.93 \pm 0.20$ (RCA $1.02 \pm 0.24$, LAD $0.94 \pm 0.18$, LCX $0.83 \pm 0.13$ ). The mean max heart rate during examination was $57 \mathrm{bpm}$ and Agatston score was $112 \pm 505$. The CT dose index and DLP for all dynamic CCTA scan were 22.9 \pm 7.0 $\mathrm{mGy}$ and $324 \pm 107 \mathrm{mGy} . \mathrm{cm}$, respectively. In total, 337 vessels were analyzed, with 35 vessels were excluded because of Chronic total occlusion, stent implants, and diffuse circumferential advanced calcification.

\section{Comparison of rCFI by plaque characteristics}

Of the 337 total vessels, 169 vessels (50.1\%) were diagnosed as normal, 99 vessels (29.3\%) as noncalcified plaques, and 69 vessels $(20.5 \%)$ as calcified plaques. The non-calcified group contained 16 vessels with positive remodeling. $\mathrm{rCF}$ for vessels with non-calcified plaques was significantly lower than that for normal vessels $(0.73 \pm 0.23$ vs. $0.88 \pm 0.22, p<0.00001)$ (Fig. 2$)$.

There was no significant difference between normal and calcified plaques $(0.88 \pm 0.22$ vs. $0.81 \pm 0.28$, $p=0.098)$, and between non-calcified and calcified plaques $(p=0.173)$. For vessels with non-calcified plaques, rCFI for those with positive remodeling was significantly lower than those without it $(0.70 \pm 0.17$ vs. $0.93 \pm 0.20, p=0.005)$. Positive remodeling was seen in three of RCA, nine of LAD and four of LCX. Similar trends in rCFI value were observed when analyzing each coronary artery (Table 3 ).

Comparison of rCFI by stenosis severity

Of the 337 total vessels, 75 vessels $(22.3 \%)$ had moderate stenosis $(50 \%-75 \%)$, and 25 vessels $(7.4 \%)$ had severe stenosis $(>75 \%)$. No stenosis and mild stenosis $(<50 \%)$ was observed in 237 vessels $(70 \%)$. $\mathrm{rCFI}$ values for moderate and severe stenoses were significantly lower than those for no and mild stenoses ( $<50 \%$ vs. $50-75 \%, 0.88 \pm 0.22$ vs. $0.73 \pm 0.24, p<0.0001 ;<50 \%$ vs $>75 \%, 0.88 \pm 0.22$ vs $0.67 \pm 0.26, p=0.00042$ ) (Fig. 3). There was no significant difference between moderate and severe stenosis

For no stenosis and mild to moderate stenosis $(n=312)$, rCFI was significantly lower for non-calcified plaques than for normal vessels $(0.76 \pm 0.23$ vs. $0.89 \pm 0.20, p<0.0001)$ (Fig. 2$)$.

\section{The potential use of rCFI in the detection of ischemia}

For the assessment of functional ischemia, 43 patients (34.6\%) underwent stress/rest MPS. The median interval between the CCTA and MPS was 60 days. 14 coronary territories were excluded according to the exclusion criteria of stent implant or severe calcifications. The remaining 115 coronary territories were 
analyzed. Of the 115 vessels, 13 territories (11.3\%) were positive for ischemia, while 102 territories (88.6\%) were negative.

rCFI was significantly lower for ischemia than non-ischemia $(0.63 \pm 0.24$ vs. $0.84 \pm 0.19, p<0.05)$ (Fig. 4).

\section{Association with rCFI and coronary risk factors}

In this study, coronary risk factors were observed in some patients, with 39 having (31.4\%) diabetes, 70 $(56.4 \%)$ having hyperlipidemia, and 68 having $(54.8 \%)$ hypertension. rCFI values were significantly lower for patients with these risk factors than those without (Table 4).

The use of the cutoff of rCFI value of 0.59 could be predictive of ischemia, with an AUC of 0.73 , a sensitivity of $53 \%$ and a specificity of $93 \%$. A representative case is presented in Figure $5 A, 5 B, 5 C$, and $5 \mathrm{D}$.

\section{Discussion}

This is the first study investigating the ability of rCFI derived from dynamic CCTA and MCIP in detecting plaque burden and myocardial ischemia. This study modifies the previous test injection method ${ }^{9}$ and attempts a new scanning protocol that can simultaneously acquire both standard CCTA and dynamic data. With this, the contrast of the coronary artery is sufficiently obtained, and intracoronary dynamic flow of contrast media-which had its starting peak CT value during the dynamic series-can be measured with good reproducibility.

rCFI naturally decreased with the degree of narrowing of the coronary lumen (Fig. 3). One interesting observation is that the presence of non-calcified plaques reduced rCFI in non-stenosis and mild to moderate coronary stenosis (Fig. 2). In addition, positive remodeling-a characteristic of high-risk plaque -also reduced rCFI. We consider the following pathophysiological phenomena as reasons for noncalcified plaques and decreased rCFl: Firstly, in fluid dynamics, increased vascular resistance in functional stenosis with non-calcified plaques causes decreased perfusion pressure at distal sites ${ }^{9}$ : Secondly, intimal proliferation in atherosclerosis reduces shear stress to the vessel wall, resulting in a vortex and slow flow ${ }^{16}$. High-risk plaques are associated with the presence of FFR-verified ischemia regardless of the degree of luminal narrowing ${ }^{17,18}$. High-risk plaques are prone to result in acute coronary syndrome and have described as strong predictors of adverse events ${ }^{19-21}$. The results of rCFI confirm that the presence of high-risk plaques predisposes the patients to ischemia and acute coronary syndrome if triggered. In contrast, there was no decrease in rCFI observed in calcified plaques. The average Agatston score for all patients was 112 , which is quite low. This may be due to having only a few subjects at the end stage of atherosclerosis after the exclusion of severe calcification cases was done. Myocardial PET studies have reported that calcified plaques do not contribute to reducing myocardial flow reserve ${ }^{22}$, which was consistent with our results. 
Ischemic territories depicted by SPECT had a significantly lower value in rCFI than non-ischemic territories (Fig. 4). When the optimal cutoff of rCFI was used to diagnose ischemia, the sensitivity was only $53 \%$, but the specificity was $93 \%$. One of the reasons for the low sensitivity is the presence of false positives in the inferior wall of the left ventricle on SPECT; that is, in the RCA territory ${ }^{23}$. In our study, the rCFI for the RCA ischemia depicted by SPECT $(n=7)$ was $0.81 \pm 0.21$. In contrast, the rCFI for LAD and LCX ischemia $(n=$ 6 ) was $0.43 \pm 0.21$. There was a discrepancy between the RCA territory and the other territories. Accordingly, SPECT diagnosis of the RCA territory may include false positives. Under hyperemic conditions, there is no decrease in coronary flow at about $40 \%$ stenosis compared to that at rest, but a significant decrease in coronary flow appears at stenosis of $80 \%$ or more ${ }^{24}$. Although rCFI is a resting flow index, it is highly specific for the detection of ischemia as a functional index-combining plaque burden and morphological stenosis.

For the analysis by patient-regardless of coronary stenosis-rCFI was significantly lower for patients with hypertension, hyperlipidemia, and diabetes than those without these conditions (Table 4). These coronary risk factors are known to affect vascular endothelial function and coronary microcirculation 25 . We speculate that rCFI may reflect vascular endothelial function before causing coronary stenosis.

The limitation of our study should be acknowledged. First, this was a single-center cohort study with a small sample size. However, there was no bias in patient selection because of the prospective study design. FFR-CT is most commonly used as a non-invasive technique using CCTA for functional coronary artery stenosis ${ }^{6,7}$. Compared to FFR-CT, extra radiation exposure should be noted because a dynamic scan was required. In this CT protocol, the average DLP was $324 \mathrm{mGy} . \mathrm{cm}$. This is considered within the diagnostic reference level of CCTA in Western countries. Meanwhile, FFR-CT requires that CT data be sent to the technology provider, as well as supercomputing $\operatorname{spec}^{6,7}$. Our image analysis did not require supercomputers and could be analyzed immediately after imaging in a relatively short time (around 30 minutes). The workflow, from the start of inspection to diagnosis, ends in one day. Finally, rCFI should be compared with invasive iFR and FFR. In this study, only six patients were compared with invasive iFR in this study, the Pearson correlation coefficient between rCFI and invasive iFR was 0.47 , indicating a moderate correlation. This presents an opportunity for the future studies.

In conclusion, the present study proposes the use of rCFI derived from dynamic CCTA as a non-invasive quantification of coronary flow dynamics. rCFI can be used for the detection of plaque burden and myocardial ischemia in stable CAD. This novel technique can be added to routine clinical workups to predict hemodynamically significant coronary stenosis in patients.

\section{Declarations}

\section{Competing interests}

The authors declare no competing interests. 


\section{Data availability}

The datasets generated during and/or analyzed during the current study are available from the corresponding author on reasonable request.

\section{References}

1 Bech, G. J. et al. Fractional flow reserve to determine the appropriateness of angioplasty in moderate coronary stenosis: a randomized trial. Circulation 103, 2928-2934, doi:10.1161/01.cir.103.24.2928 (2001).

2 Tonino, P. A. et al. Fractional flow reserve versus angiography for guiding percutaneous coronary intervention. The New England journal of medicine 360, 213-224, doi:10.1056/NEJMoa0807611 (2009).

3 Neumann, F. J. et al. 2018 ESC/EACTS Guidelines on myocardial revascularization. Eurolntervention : journal of EuroPCR in collaboration with the Working Group on Interventional Cardiology of the European Society of Cardiology 14, 1435-1534, doi:10.4244/eijy19m01_01 (2019).

4 Pijls, N. H. et al. Measurement of fractional flow reserve to assess the functional severity of coronary-artery stenoses. The New England journal of medicine 334, 1703-1708, doi:10.1056/nejm199606273342604 (1996).

5 Sen, S. et al. Development and validation of a new adenosine-independent index of stenosis severity from coronary wave-intensity analysis: results of the ADVISE (ADenosine Vasodilator Independent Stenosis Evaluation) study. Journal of the American College of Cardiology 59, 1392-1402, doi:10.1016/j.jacc.2011.11.003 (2012).

6 Min, J. K. et al. Diagnostic accuracy of fractional flow reserve from anatomic CT angiography. Jama 308, 1237-1245, doi:10.1001/2012.jama.11274 (2012).

7 Norgaard, B. L. et al. Diagnostic performance of noninvasive fractional flow reserve derived from coronary computed tomography angiography in suspected coronary artery disease: the NXT trial (Analysis of Coronary Blood Flow Using CT Angiography: Next Steps). Journal of the American College of Cardiology 63, 1145-1155, doi:10.1016/j.jacc.2013.11.043 (2014).

8 Motoyama, S. et al. Multislice computed tomographic characteristics of coronary lesions in acute coronary syndromes. Journal of the American College of Cardiology 50, 319-326, doi:10.1016/j.jacc.2007.03.044 (2007).

9 Nagao, M. et al. Quantification of coronary flow using dynamic angiography with 320-detector row CT and motion coherence image processing: Detection of ischemia for intermediate coronary stenosis. European journal of radiology 85, 996-1003, doi:10.1016/j.ejrad.2016.02.027 (2016). 
Shimomiya, Y. et al. Dynamic flow imaging using 320-detector row CT and motion coherence analysis in coronary aneurysms associated with Kawasaki disease. Cardiology in the young 28, 416-420, doi:10.1017/s1047951117002293 (2018).

11 Jessen, K. A., Shrimpton, P. C., Geleijns, J., Panzer, W. \& Tosi, G. Dosimetry for optimisation of patient protection in computed tomography. Applied radiation and isotopes : including data, instrumentation and methods for use in agriculture, industry and medicine 50, 165-172, doi:10.1016/s0969-8043(98)00024-4 (1999).

12 Shiina, Y., Inai, K., Takahashi, T., Shimomiya, Y. \& Nagao, M. Clinical impact of cardiac computed tomography derived three-dimensional strain for adult congenital heart disease: a pilot study. The international journal of cardiovascular imaging 36, 131-140, doi:10.1007/s10554-019-01691-w (2020).

13 Berman, D. S. et al. Roles of nuclear cardiology, cardiac computed tomography, and cardiac magnetic resonance: assessment of patients with suspected coronary artery disease. Journal of nuclear medicine : official publication, Society of Nuclear Medicine 47, 74-82 (2006).

14 Hachamovitch, R. et al. Prognostic implications of myocardial perfusion single-photon emission computed tomography in the elderly. Circulation 120, 2197-2206, doi:10.1161/circulationaha.108.817387 (2009).

15 Klocke, F. J. et al. ACC/AHA/ASNC guidelines for the clinical use of cardiac radionuclide imaging-executive summary: a report of the American College of Cardiology/American Heart Association Task Force on Practice Guidelines (ACC/AHA/ASNC Committee to Revise the 1995 Guidelines for the Clinical Use of Cardiac Radionuclide Imaging). Journal of the American College of Cardiology 42, 13181333, doi:10.1016/j.jacc.2003.08.011 (2003).

16 Gibson, C. M. et al. Relation of vessel wall shear stress to atherosclerosis progression in human coronary arteries. Arteriosclerosis and thrombosis : a journal of vascular biology 13, 310-315, doi:10.1161/01.atv.13.2.310 (1993).

17 Gaur, S. et al. Coronary plaque quantification and fractional flow reserve by coronary computed tomography angiography identify ischaemia-causing lesions. European heart journa/ 37, 1220-1227, doi:10.1093/eurheartj/ehv690 (2016).

18 Park, H. B. et al. Atherosclerotic plaque characteristics by CT angiography identify coronary lesions that cause ischemia: a direct comparison to fractional flow reserve. JACC. Cardiovascular imaging 8, 1-10, doi:10.1016/j.jcmg.2014.11.002 (2015).

19 Chang, H. J. et al. Coronary Atherosclerotic Precursors of Acute Coronary Syndromes. Journal of the American College of Cardiology 71, 2511-2522, doi:10.1016/j.jacc.2018.02.079 (2018). 
20 Motoyama, S. et al. Computed tomographic angiography characteristics of atherosclerotic plaques subsequently resulting in acute coronary syndrome. Journal of the American College of Cardiology 54, 49-57, doi:10.1016/j.jacc.2009.02.068 (2009).

21 Driessen, R. S. et al. Adverse Plaque Characteristics Relate More Strongly With Hyperemic Fractional Flow Reserve and Instantaneous Wave-Free Ratio Than With Resting Instantaneous Wave-Free Ratio. JACC. Cardiovascular imaging 13, 746-756, doi:10.1016/j.jcmg.2019.06.013 (2020).

22 Naya, M. et al. Quantitative relationship between the extent and morphology of coronary atherosclerotic plaque and downstream myocardial perfusion. Journal of the American College of Cardiology 58, 1807-1816, doi:10.1016/j.jacc.2011.06.051 (2011).

23 Malik, A. O. et al. Significance of inferior wall ischemia in non-dominant right coronary artery anatomy. World journal of cardiology 9, 261-267, doi:10.4330/wjc.v9.i3.261 (2017).

24 Uren, N. G. et al. Relation between myocardial blood flow and the severity of coronary-artery stenosis. The New England journal of medicine 330, 1782-1788, doi:10.1056/nejm199406233302503 (1994).

25 Rafieian-Kopaei, M., Setorki, M., Doudi, M., Baradaran, A. \& Nasri, H. Atherosclerosis: process, indicators, risk factors and new hopes. International journal of preventive medicine 5, 927-946 (2014).

\section{Tables}

Due to technical limitations, the tables are only available as a download in the supplemental files section.

\section{Figures}




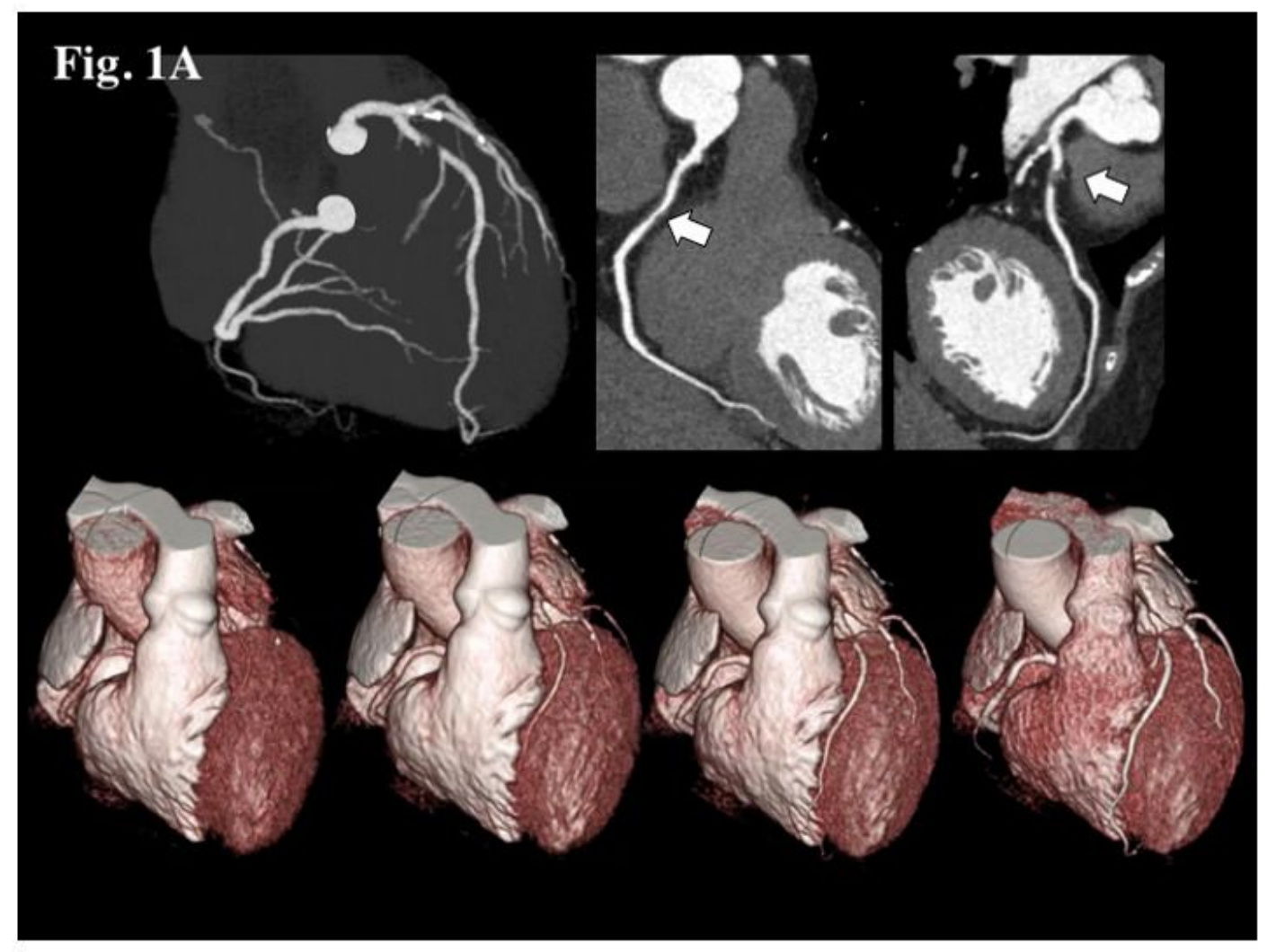

Fig. 1B

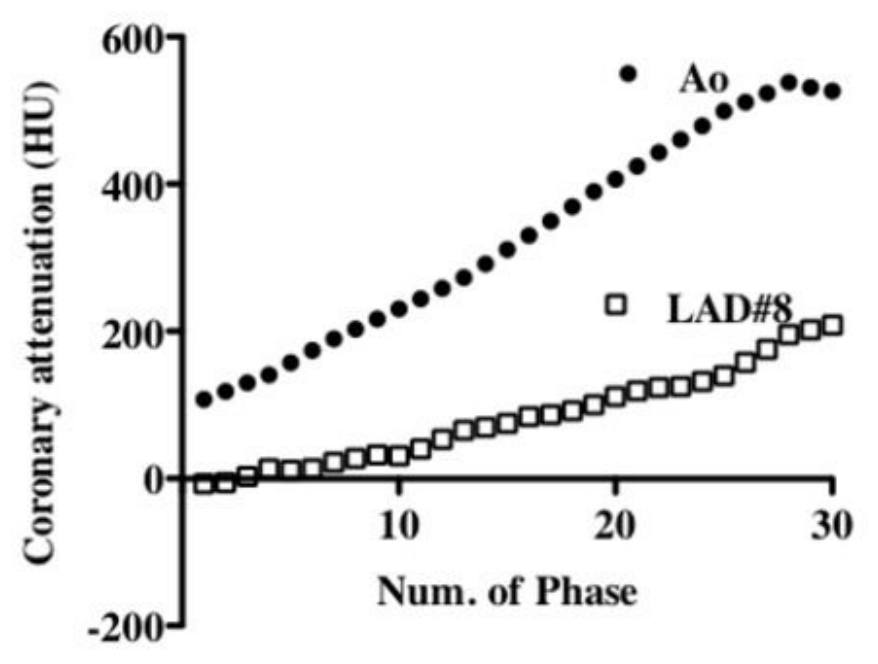

Figure 1

A. A woman in her 60s with an effort angina. CCTA at boost scan (upper row) shows a moderate stenosis with non-calcified plaque at RCA \#2 (arrow) and $90 \%$ or more stenosis with non-calcified plaque at LAD \#6 (arrow). Volume rendering whole heart dynamic CCTA reconstructed by motion coherence image processing (lower row) demonstrates a first pass of contrast media in coronary arteries with a high spatial resolution and noise reduction. B. Calculation of rCFI Graphs for the same patient as in Figure 1 
show time-density curves of intra-coronary attenuation ( $\mathrm{HU}$; Hounsfield units) for the ascending aorta and distal portion of the LAD \#8 throughout all phases. The maximum upslope (HU/phase) for the sites was calculated from the liner upslope of the CT value, which represents the pass of contrast media. From 0 to the 28th phase, the maximum upslope for the ascending aorta and distal site of stenosis were 17.7 $\mathrm{HU} /$ phase and $8.2 \mathrm{HU} /$ phase. According to the maximum upslope method, the RCFI of LAD was calculated as $0.46(8.2 / 17.7)$.
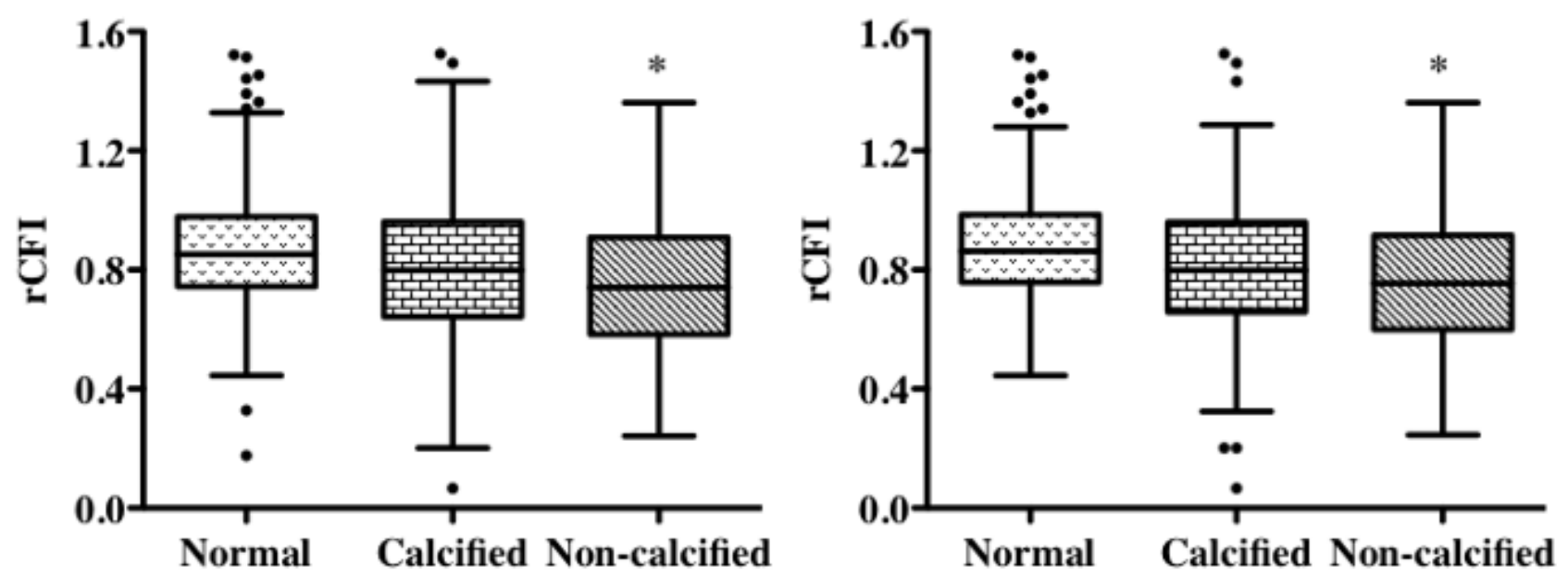

Figure 2

Comparison of rCFI by plaque characteristics The left shows Box-whisker plot for all 337 arteries, and the right shows for arteries with 0 to $75 \%$ stenosis excluding $75 \%$ or more stenosis. For all 337 arteries and 312 arteries with 0 to $75 \%$ stenosis, rCFI for the non-calcified plaque was significantly lower than normal. There was no difference between non-calcified plaque and calcified plaque and between normal and calcified plaque. *: $p<0.0001$ The horizontal lines indicate maximum and minimum values, and the box indicates 25 and 75 percentiles. 


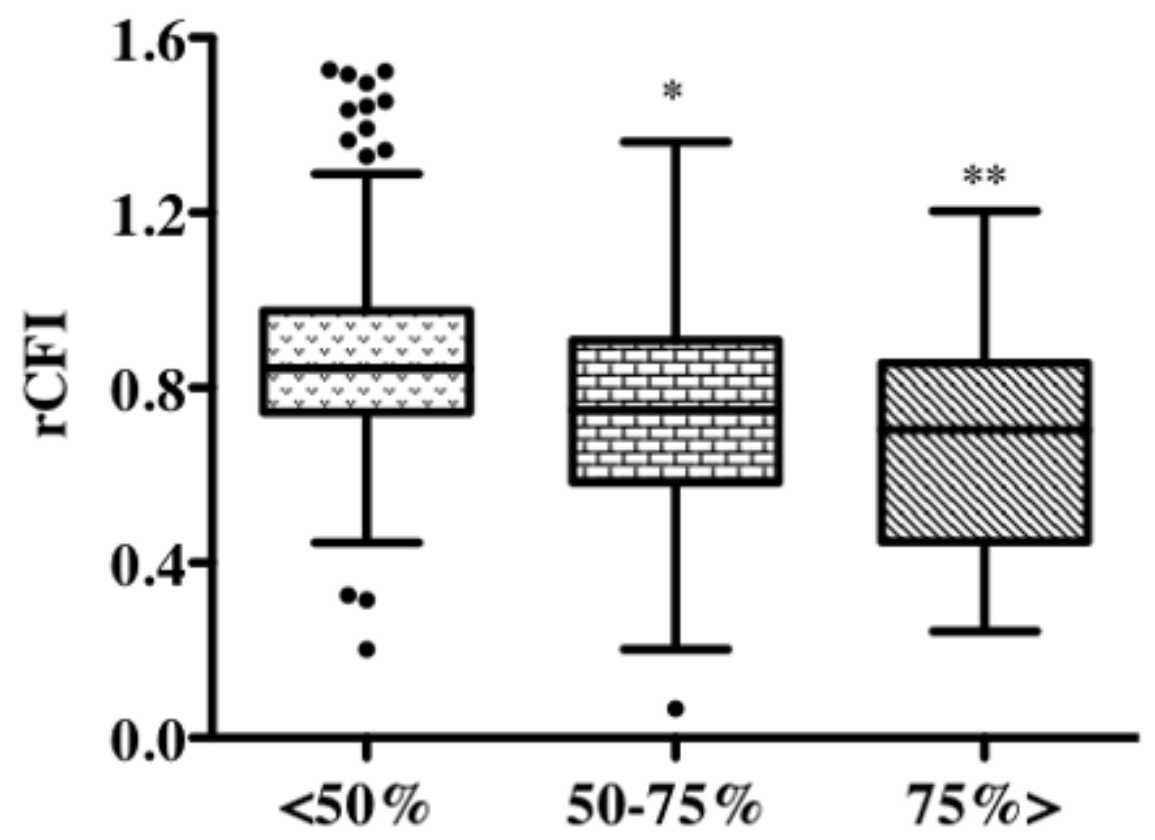

Figure 3

Comparison of rCFI by stenosis severity Box-whisker plot for all 337 arteries demonstrates that rCFI for arteries with $50-75 \%$ stenosis and $\geq 75 \%$ stenosis was significantly lower than that for arteries with $<50 \%$ stenosis. *: $<50 \%$ vs. $50-75 \%, p<0.0001 ; * *:<50 \%$ vs $>75 \%, p=0.00042$ The lines and box are the same as Figure 2.

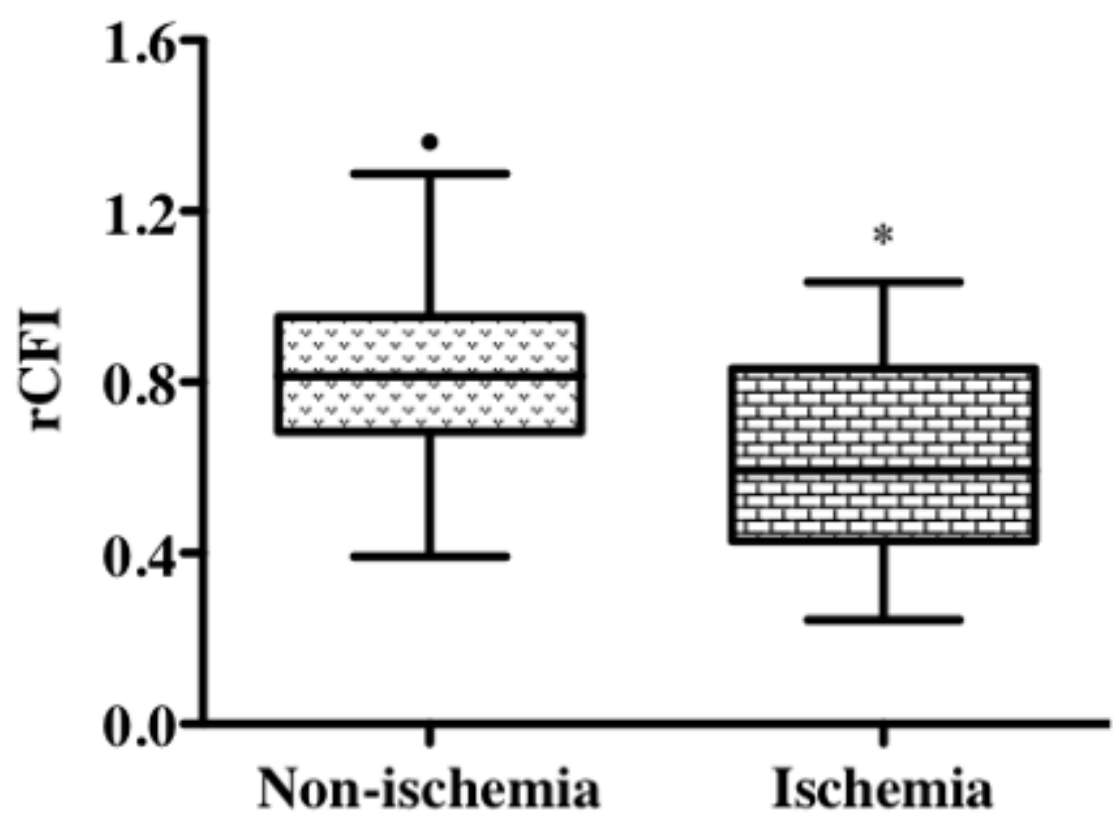




\section{Figure 4}

Comparison of rCFI between non-ischemia and ischemia Box-whisker plot of 115 coronary territories shows that rCFI was significantly lower for ischemia than non-ischemia. *: $p<0.05$ The lines and box are the same as Figure 2.

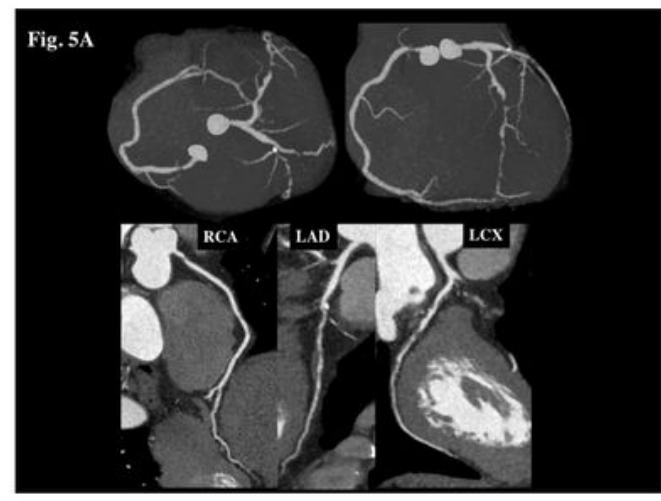

\section{Fig. 5B}
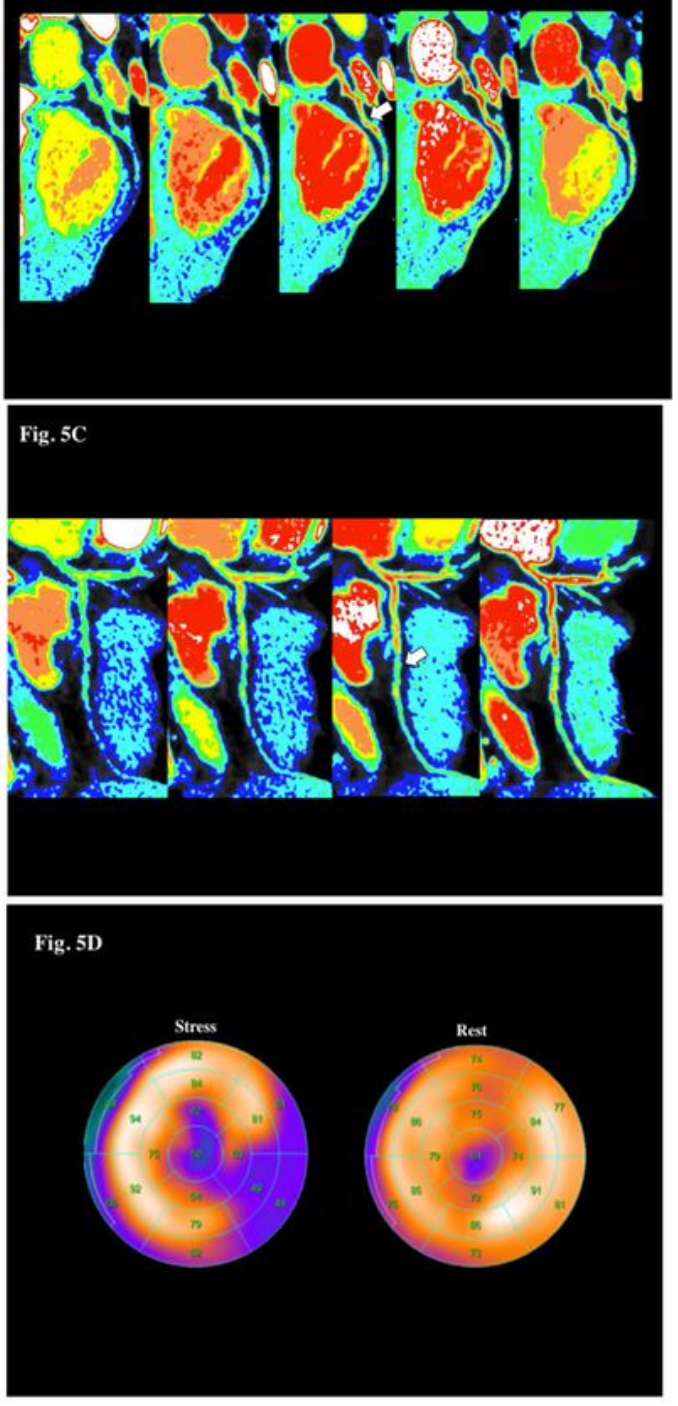

Figure 5 
A man in his 40s with effort angina A: Angiographic views of CCTA show significant stenoses at multivessels and a small calcification of proximal LAD (upper row). Curved MPR images show a moderate stenosis with non-calcified plaque at RCA \#1 and diffuse moderate to severe stenoses with non-calcified plaque at LAD \#7 and LCX (lower row). B: Dynamic color-coded images of LAD According to intracoronary attenuation, the coronary artery was delineated to 5 color scales. Warm colors represent high CT values, and cold colors represent low CT values. Dynamic color-coding can visualise a first pass of contrast media in the $L A D$, and demonstrates high attenuation without delay at the distal site of stenosis (arrow). rCFI of LAD is 0.71 . C: Dynamic color-coding of LCX shows low attenuation with delay at the distal site of stenosis (arrow). rCFI of LCX is 0.39. D: Myocardial perfusion maps represent decreased uptake ratio in the LCX territory at stress (left) and normal uptake at rest (right). The presence of ischemia in the LCX territory is consistent with decrease rCFI.

\section{Supplementary Files}

This is a list of supplementary files associated with this preprint. Click to download.

- Table1.tiff

- Table2.tiff

- Table3.tiff

- Table4.tiff

- SupplementFig.1A.mp4 